\title{
Surface energy controlled $\alpha-\gamma-\alpha$ transformation texture and microstructure character study in ULC steels alloyed with $\mathrm{Mn}$ and $\mathrm{Al}$
}

\author{
Jai Gautam · Roumen Petrov $\cdot$ Leo Kestens • \\ Elke Leunis
}

Received: 3 August 2007/ Accepted: 1 November 2007/Published online: 13 March 2008

(C) The Author(s) 2008

\begin{abstract}
In this article, an ultralow-carbon steel grade alloyed with $\mathrm{Mn}$ and $\mathrm{Al}$ has been investigated during $\alpha-\gamma-\alpha$ transformation annealing in vacuum. Typical texture and microstructure has evolved as a monolayer of grains on the outer surface of transformation-annealed sheets. This monolayer consists of $<100>/ / \mathrm{ND}$ and $<110>/ / \mathrm{ND}$ fibre, which is very different from the bulk texture components. The selective driving force is believed to reside in the anisotropy of surface energy at the metal-vapour interface. The grain morphology is very different from the bulk grains. Moreover, $30-40 \%$ of the grain boundary interfaces observed in the RD-TD surface sections are tilt incoherent $<110>$ $70.5^{\circ}$ boundaries, which are known to exhibit reduced interface energy. Hence, the conclusion can be drawn that the orientation selection of surface grains is strongly controlled by minimization of the interface energy; both metal/vapour and metal/metal interfaces play a roll in this.
\end{abstract}

\section{Introduction}

It is well known that surface energy anisotropy is the driving force for the orientation selection at the metalvapour interface. This impacts the microstructure and

J. Gautam · R. Petrov

Department of Metallurgy and Materials Science,

Ghent University, Technologiepark 903, Ghent 9052, Belgium

J. Gautam $(\bowtie) \cdot$ R. Petrov $\cdot$ L. Kestens

Department of Material Science and Engineering,

Delft University of Technology, Mekelweg 2, 2628 Delft,

$\mathrm{CD}$, The Netherlands

e-mail: j.p.gautam@tudelft.nl

E. Leunis

OCAS N.V, Arcelor Mittal Research Industry, Ghent, Belgium texture evolution at surface during phase transformation. Although the exact value of the interfacial energy between the atmosphere and the steel surfaces is largely unknown, it is widely accepted that the bcc $\{100\}$ and $\{110\}$ planes have a relatively low surface energy [1-5].

The austenite-to-ferrite phase transformation, which is an inherent feature of low-alloyed low-carbon steels, has scarcely been investigated for developing surface textures. Hashimoto et al. [6, 7] investigated the $\alpha-\gamma-\alpha$ phase transformation texture at the surface of an ultralow-carbon cold-rolled steel sheet and reported that a $<100>/ / N D$ texture was formed rather than the usual $<111>/ / N D$ texture. They interpreted this experimental result by assuming that the elastic work in the ND direction associated with the transformation could be larger than that in any other direction. Aspeden et al. [2] reported that an annealing treatment in the austenite phase followed by slow cooling in ultralow-carbon steel resulted in a stronger $<100>/ / \mathrm{ND}$ texture. They assumed this due to the fact that this surface texture leads to the lowest surface energy. The $<100>/$ /ND surface grains nucleate more rapidly because they have the lowest interfacial energy with the surrounding atmosphere. Tomida and Tanaka [3] clarified that an extremely sharp $<100>/$ /ND texture can be formed at the surface of Mnalloyed low-carbon steel by means of an inter-critical annealing or a heat treatment in the $\gamma$ region.

The surface textures reported in the literature do not include a precise description of the grain morphology and grain structure at the surface. In a recent study [5], the present author has reported a monolayer of surface grains after a short phase transformation annealing which produces a surface texture with $\{100\}$ and $\{110\}$ orientations very different from the conventional $\{111\}$ bulk texture. In order to interpret the surface effect two alternative mechanisms were considered: an orientation selection 
mechanism controlled by surface energy [1-7] or alternatively a variant selection mechanism resulting from the release of transformation stresses along the normal direction of the sheet $[6,7]$.

Based on the following grounds it was concluded that the surface energy mechanism is responsible for the surface texture development: (i) the observed $\{001\}$ and $\{110\}$ orientations correspond to the low-energy vapour/solid crystal interfaces which are generally observed for bcc metals and (ii) these orientations are only present as a shallow monolayer of grains whereas the residual stress argument would rather match with a gradual orientation gradient in the vicinity of the surface. In the present article, the morphological and crystallographic nature of the monolayer of $\{001\}-\{110\}$ grains will be further explored and compared with the features of bulk metal grains.

\section{Experimental}

Two alloys, denominated L2 and L3, with the chemical composition listed in Table 1 were hot-rolled above the Ar3 temperature, air cooled and annealed at $700{ }^{\circ} \mathrm{C}$ in order to simulate the slow cooling trajectory of the industrial hot band coiling process. After surface oxide removal (pickling) the hot-rolled sheets were cold-rolled to a $70 \%$ reduction. The cold-rolled material was annealed in the austenite region for 2-3 min as shown in Fig. 1. An infrared furnace was used for this heat treatment. During annealing the furnace atmosphere was slightly reducing with a $5 \% \mathrm{H}_{2}-95 \% \mathrm{~N}_{2}$ gas composition. In order to know the critical temperatures, various heating and cooling experiments were performed in a dilatometer and the critical temperatures were derived from the dilatation curves, cf. Table 2.

Table 1 Chemical composition

\begin{tabular}{lllll}
\hline Wt $(\%)$ & $\mathrm{C}$ & $\mathrm{Mn}$ & $\mathrm{Si}$ & $\mathrm{Al}$ \\
\hline $\mathrm{L} 2$ & 0.002 & 1.28 & 0.22 & 0.29 \\
L3 & 0.003 & 0.75 & 0.23 & 0.12 \\
\hline
\end{tabular}

Table 2 Critical temperatures

\begin{tabular}{llr}
\hline & L2 & \multicolumn{1}{c}{ L3 } \\
\hline Ac1 $\left({ }^{\circ} \mathrm{C}\right)$ & 940 & 949 \\
$\operatorname{Ac} 3\left({ }^{\circ} \mathrm{C}\right)$ & 990 & 1,000 \\
$\operatorname{Ar} 1\left({ }^{\circ} \mathrm{C}\right)$ & 832 & 845 \\
$\operatorname{Ar} 3\left({ }^{\circ} \mathrm{C}\right)$ & 884 & 910 \\
\hline
\end{tabular}

Material characterization

First, the surface textures were measured by X-ray texture goniometer coupled with molybdenum crystal, on the clean cold-rolled sheets. The diffraction data were received from the depth of @8 microns underneath the sheet surface. Afterwards these samples were subjected to the transformation annealing treatment and cooled to room temperature. After annealing, the XRD texture measurement was again performed on the sample surface. The conventional X-ray diffraction method was used to measure the $\{100\},\{110\},\{211\}$ and $\{310\}$ pole figures. The orientation distribution function (ODF) was determined using software developed by Van Houtte [8]. The through thickness microstructure was examined with optical microscopy. The scanning electron microscope Phillips XL-30 with integrated EBSD system was used to perform orientation imaging microscopy (OIM) to characterize the cross-sectional texture gradient as well as the surface of the samples to study the nature of the grains and their morphology. TSL software was used for the analysis of grain orientation data.

Serial sectioning of RD-TD surface:

In an attempt to determine the grain boundary character distribution serial sectioning was carried out. The RD-TD surface of the transformation-annealed sheet was mechanically polished using 1-micron cloth wheel with fine grain silica solution. During polishing the sheet thickness was regularly measured with a screw gauge with a precision of $1 \mu \mathrm{m}$. The RD-TD surface cross sections were examined
Fig. 1 Schematic of transformation annealing and sample section for X-ray texture analysis

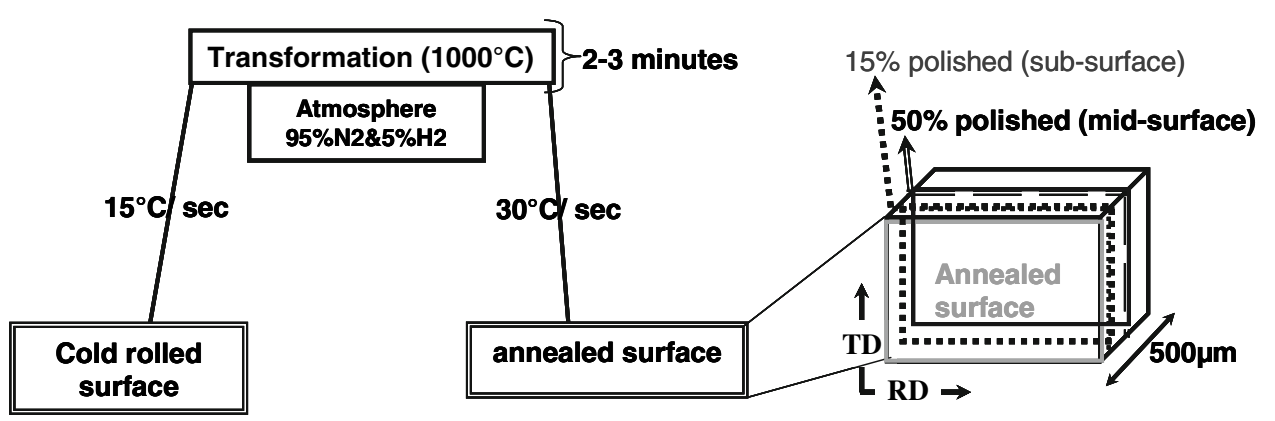


in scanning electron microscope and orientation contrast microscopy was performed.

\section{Results}

Microstructure and X-ray texture after transformation annealing

Results obtained after transformation annealing are shown in Fig. 2. They are similar for both alloys. The microstructures after transformation annealing show completely transformed grain through thickness of both alloys L2 and L3. The surface textures of the cold-rolled samples observed by XRD exhibits the characteristic $\alpha$ and $\gamma$ fibres. After transformation annealing the surface textures revealed a $<100>$ and $<110>$ fibre with an intensity of twice the random. After removing the surface by mechanical polishing, the subsurface and mid-surface were examined again in the X-ray. The ODFs display a transformed $\gamma$ fibre at the subsurface $(15 \%)$ and the mid-layer $(50 \%)$ with increase in intensity with seven to eight times of random which is very different from surface texture components. The central zone texture in the annealed sheet shows strong gamma fibre after transformation. This could be due to a texture memory effect normally observed in low-carbon steel and manganese is known to enhance this effect [9].

Through thickness grain morphology and texture gradient

Orientation imaging microscopy was carried out on the RD-ND sections of the samples with a surface texture consisting of a $<100>$ and $<110>$ fibre. This was done in order to reveal microstructural and/or textural gradients across the thickness of the sheet. Figure 3 shows the inverse pole figure map in grey scale. A single layer of surface grain with specific texture (predominantly $<100>$ and $<110>$ fibre grains) and morphology (elongated along $\mathrm{RD})$ is present in both the samples. It is noticed that material L2 displays thinner layer of surface grains compared to the material L3 with lower Mn and Al content.
Fig. 2 The transformation microstructures and X-ray textures on the RD-TD surface and underneath
Fig. 3 Inverse pole figure map in grey scale observed after $(\alpha-\gamma-\alpha)$ transformation in the RD-ND plane for materials L2 and $\mathrm{L} 3$
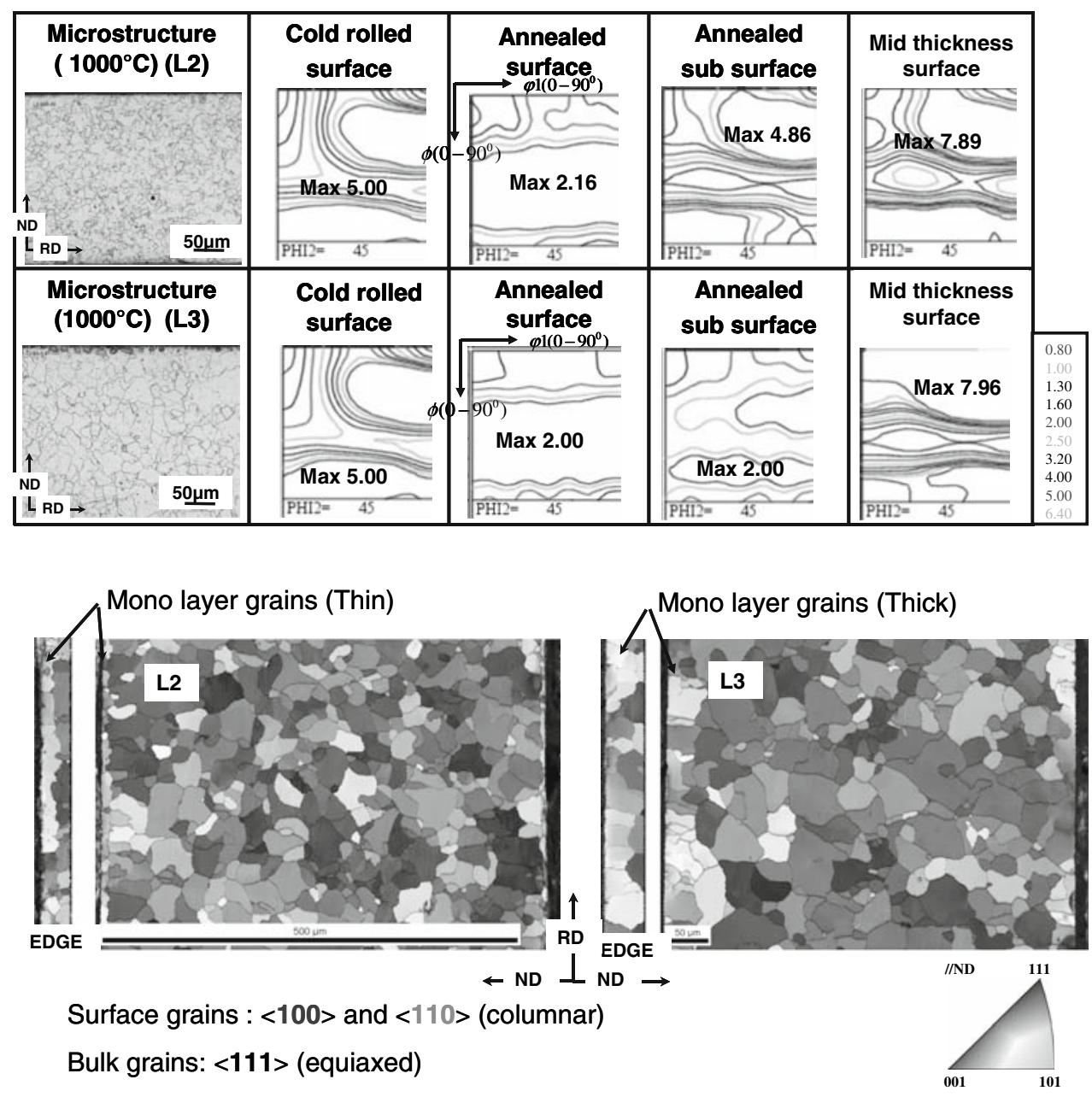
Grain morphology and grain boundary character at the surface

Figure 5 shows the inverse pole figure maps as well as high-angle grain boundary maps at 5 and 18 microns depth from the surface. The grain morphology at 3\% depth is very different from the texture below. The grains at the surface are larger and the shape is very irregular, while deeper in the material the grains are much more regular in shape and size.

A high-angle grain boundary map was plotted where the criterion of rotation angle was minimum $15^{\circ}$ to $180^{\circ}$ maximum. An overlay of Sigma 3 boundaries was made on the high-angle grain boundary map as shown in Fig. 4 below. The grain boundaries at the surface have a much larger fraction $( \pm 40 \%)$ of low-energy boundaries (sigma 3 ) compared to the bulk grains ( $\pm 10 \%$ of sigma boundaries). Both alloys L2 and L3 have a similar behaviour.

Coincident site lattice (CSL) boundaries have a given fraction of atoms in the grain boundary plane, which are coincident to both lattices separated by the grain boundary.
These boundaries are classified in terms of $\Sigma$ values, which denote the fraction of atoms in coincidence. Each coincidence relationship can be expressed by an axis-angle pair, which gives 24 possibilities in the cubic system, corresponding to the 24 symmetry elements [10]. Due to this crystal symmetry there are often 24 misorientations that can achieve a given $\Sigma$ value. For example $\Sigma 3$ can be achieved by a $70.53^{\circ}$ rotation about $<110>$ or $60^{\circ}$ about $<111>$ and 109.47 about [110]. In order to specify whether a boundary is a CSL boundary, a tolerance $\Delta$ is used which is given by $\Delta=K / \Sigma^{n}$. Common values are $15^{\circ}$ for $K$ and $1 / 2$ for $n$. This is the so-called Brandon criterion [11].

In an attempt to characterize the boundary character of surface monolayer grains, the CSL boundaries criterion was applied. Figure 5 shows the fraction of the different types of CSL boundaries at the surface of alloys L2 and L3. A very significant amount of $\Sigma 3$ CSL boundaries varying from $38 \%$ to $45 \%$ is noticed at the surface. It is important to note that the distribution is independent of the size of the scanned area of the monolayer grains for both the alloys L2 and L3.

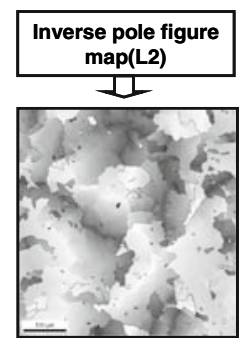

RD-TD $2^{\text {nd }}$ section

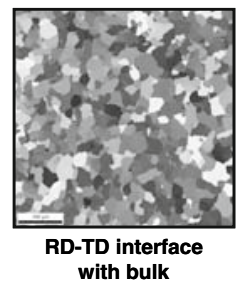

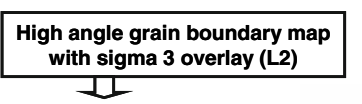
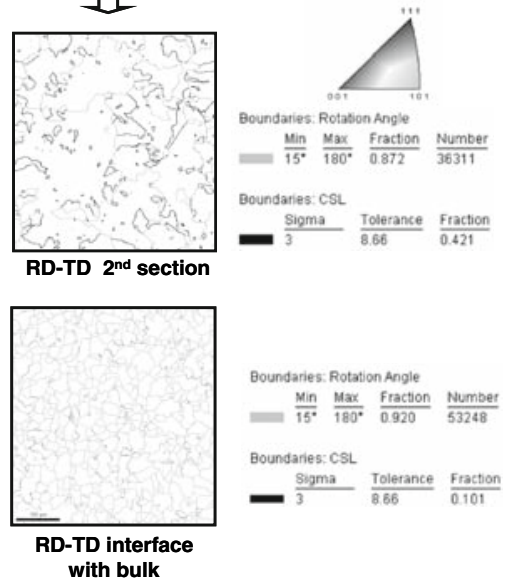

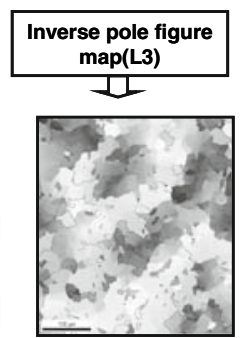

RD-TD $2^{\text {nd }}$ section

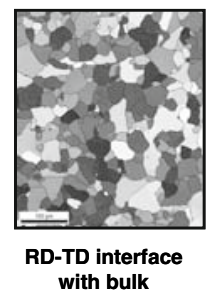

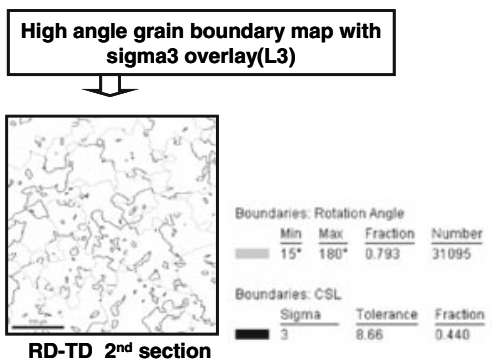

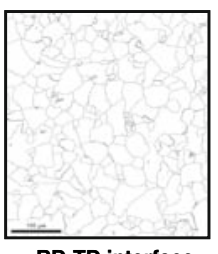

with bulk
wD interface

Fig. 4 Inverse pole figure map (grey scale) and high-angle grain boundary map with an overlay of $\Sigma 3$ boundaries in the RD-TD surface sections and in the bulk interface section of the alloys L2 and L3

Fig. 5 The fraction of CSL boundaries in different OIM scan areas in sizes at first section of RD-TD surface of alloys L2 (left) and L3 (right)
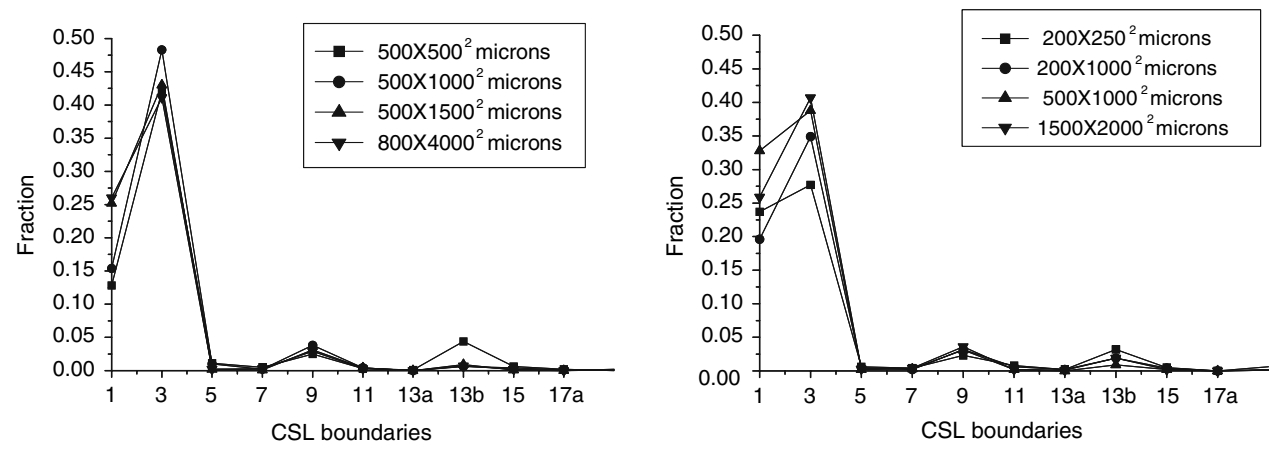


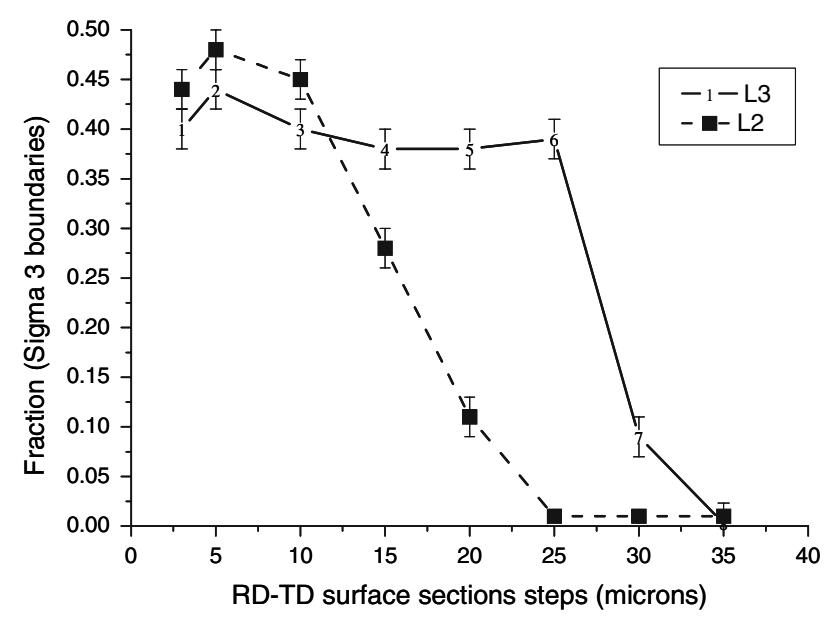

Fig. 6 The fraction of sigma 3 boundaries at different sections below the surface

CSL boundaries in function of the depth from the surface

Figure 6 shows the fraction of sigma 3 boundaries at different serial sections below the surface. Through the thickness of the monolayer the fraction of sigma 3 boundaries is rather constant. As could be noticed already on the IPF in cross section, the alloy L2 which has high amount of $\mathrm{Mn}$ and $\mathrm{Al}$ shows a thin monolayer compared to the alloy L3 with a low amount of Mn and Al.

\section{Discussion}

The $\alpha-\gamma-\alpha$ transformation microstructure of the two alloys L2 and L3 has revealed a typical surface texture with selected $\{100\}$ and $\{110\}$ planes parallel to the solid/ vapour interface. It appears that a specific orientation selection mechanism has occurred during $\alpha-\gamma-\alpha$ transformation at the cold-rolled surface. There are different explanations reported in the literature for the $<100>/ / \mathrm{ND}$ and $<110>/ / \mathrm{ND}$ texture formation at the surface, but the following two mechanisms are found to be more relevant in the present study [2-4, 6, 7]: (i) an orientation selection mechanism controlled by surface energy or (ii) a variant selection mechanism resulting from the release of transformation stresses towards the normal direction.

The first possibility assumes that the orientation selection is driven by the minimization of surface energy at the metal-vapour interface. According to the classical Wulff theory [12], the equilibrium shape of a solidifying metal volume is described by the inner envelope of the anisotropic surface energy function $E(<h k l>$ ) (with $<h k l>$ an arbitrary crystal direction). Therefore, when the surface energy plot ( $\gamma$ plots) contains sharp cusps (cf. Fig. 7) the equilibrium shape is a polyhedron with the largest facets perpendicular to the $\langle h k l\rangle$ direction corresponding to the lowest interfacial free energy [12]. The precise shape of the $E(\langle h k l\rangle)$ dependence is determined by the anisotropy of the bonding energy in the metallic crystal lattice. If only first nearest neighbour interactions are taken into account, the Wulff polyhedron of bcc iron is known to be a dodecahedron with $8\{110\}$ and $4\{100\}$ surfaces, cf. Fig. 7 [13]. The equilibrium shape was experimentally verified by evaporation and solidification of small single crystals at high temperatures in a specific annealing atmosphere [14]. It was reported that ultra-fine particles of pure iron produced by evaporation in an inert atmosphere displayed the dodecahedron shape of Fig. 7 [15].

The development of the particular surface texture during $(\alpha-\gamma-\alpha$ transformation shows some remarkable features, which may be explained by a selection mechanism on the basis of minimization of metal-vapour surface energy. First, it is noticed that the observed $\{001\}$ and $\{110\}$
Fig. 7 The equilibrium shape of bcc iron: a truncated rhombic dodecahedron consisting of 8 $\{110\}$ and $4\{100\}$ surfaces with schematic explanation
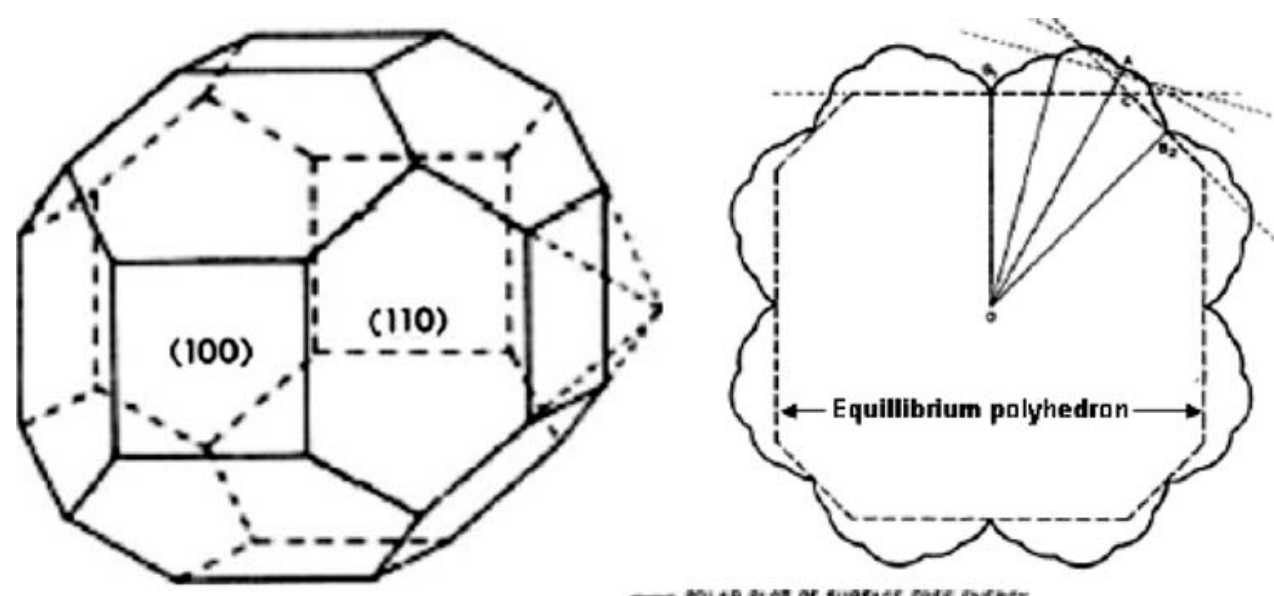

molat hot or suabace mare oviber

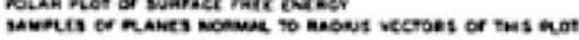


orientations precisely correspond to the free surface planes of minimum energy as predicted by the Wulff polyhedron. Second, it is observed that these particular texture components only appear at the very surface of the material, i.e. only the grains which are in direct contact with the metal/ vapour interface are of the $\{001\}$ or $\{110\}$ component, whereas the underlying layer of grains which do not possess a metal/vapour interface already exhibit a different texture.

It can also be observed that edge grains display very specific grain boundary morphology in the RD-TD surface. In contrast to the relatively regular GB morphology produced by curvature-driven growth, the surface grains display a very irregular almost acicular shape (Fig. 4). From the point of view of crystallography, the grain boundaries observed in the RD-TD surface section show the highest amount of $\Sigma 3$ CSL boundaries with the twin type axis angle relationship of $<110>70.5^{\circ} \equiv<111>60^{\circ}$. From the morphology of these GBs it is clear that they cannot be coherent twin boundaries, but even incoherent twin boundaries are known to exhibit a lower GB energy as compared to random high angle GBs [16]. Figure 6 shows the fraction of $\Sigma 3$ boundaries on various serial sections underneath the RD-TD surface. It can be observed that the surface area with a high fraction of $\Sigma 3$ GBs extends to a larger depth underneath the surface in the L3 alloy, which contains a higher amount of $\mathrm{Mn}$ and $\mathrm{Al}$ as compared to the L2 alloy. This also corresponds to the microstructural observations of Fig. 3 in which it can be seen that the columnar monolayer of surface grains stretches out over a deeper range in the L3 alloy. Both the observation of an increased incidence of low-energy $\Sigma 3$ GBs and the presence of $\{001\}$ and $\{110\}$ with a reduced solid/vapour interfacial energy seem to indicate that creation of the surface structure and texture was strongly energy-controlled. How this irregular grain shape (Fig. 4) was formed and what it means in terms of the orientation selection mechanism is not clear at this stage.

The second explanation for the presence of the $<100>$ and $\langle 110\rangle$ texture at the surface after transformation was discussed. Hasimoto et al. [6, 7] proposed the idea that variant selection during the $(\alpha-\gamma-\alpha)$ transformation is controlled by the minimization of elastic accommodation stress resulting from the volume and shape changes associated with the sequence of allotropic phase transformations. As the elastic boundary conditions of a nucleating ferrite grain in an austenite matrix are entirely different in the bulk of the sheet as compared to the surface, it is reasonable to assume that also this mechanism will give rise to a specific surface texture. Indeed, at the surface of the sheet the elastic stresses may be more easily relaxed in the ND direction as compared to the bulk of the material. Hashimoto et al. have calculated the ratio of elastic work in the normal direction to the total elastic work $\mathrm{R}_{\gamma-\alpha}(\gamma-\alpha)$ for all possible product variants, which were predicted by the $\mathrm{K}-\mathrm{S}$ orientation relations. They showed the development of an intense $<100>/ / N D$ texture after simulation of the $(\alpha-\gamma-\alpha)$ transformation under the hypothesis that $\mathbf{R}_{(\alpha-\gamma)}$ and $\mathbf{R}_{(\gamma-\alpha)}$ is not less than $35 \%$ of the total elastic work. In other words, particular variants among the 24 possible variants, which require more than $35 \%$ work along the normal direction out of the total elastic work, were predominantly selected in their simulations. The present data do not only show $\{001\}$, but also $\{110\}$ orientations, though the latter cannot be explained by the work of Hashimoto et al. Moreover, on the basis of the elastic stress relaxation model of Hashimoto et al. one would rather expect a more gradual texture gradient from the surface towards the bulk as the elastic stress relaxation along ND will gradually develop towards the surface of the sheet. The present data rather show a discontinuous change of surface texture and structure. Thus, for these reasons the present experimental results seem to confirm the surface energy model rather than the elastic stress relaxation theory of Hashimoto et al.

\section{Conclusions}

The selected alloy compositions L2 and L3 exhibit a specific surface texture after $(\alpha-\gamma-\alpha)$ transformation annealing of a cold-rolled sheet. The annealed sheet surfaces of the two alloys exhibit a monolayer of grains in direct contact with the metal/vapour interface and with predominant $<100>/ / \mathrm{ND}$ and $<110>/ / \mathrm{ND}$ fibre texture. The grain boundaries at surface have a high fraction of Sigma 3 CSL boundaries which correspond to low energy boundaries. Orientation selection on the basis of minimization of the metal/vapour interface energy seems to be the most appropriate hypothesis to explain the present results.

Acknowledgement Authors are grateful to OCAS N.V., Arcelor Mittal Research Industry, Ghent, Belgium for the financial support.

Open Access This article is distributed under the terms of the Creative Commons Attribution Noncommercial License which permits any noncommercial use, distribution, and reproduction in any medium, provided the original author(s) and source are credited.

\section{References}

1. Dunn CG, Walter J (1962) Trans Met Soc AIME 224:518

2. Aspeden RG, Berger J, Trout HE (1968) Acta Metall 16:1027

3. Tomida T, Tanaka (1995) ISIJ International 35:548

4. Yoshinaga N, Kestens L, De Cooman BC (2005) Mater Sci Forum 495-497:1267 
5. Gautam J, Petrov R, Kestens L (2007) Mater Sci Forum 550: 503

6. Hashimoto O, Satoh S, Tanaka T (1980) Tetsu-to-hagane 66:102

7. Hashimoto O, Satoh S, Tanaka T (1980) Tetsu-to-hagane 66:112

8. Van Houtte P (1995) The MTM-FHM software system version 2 users manual

9. Yoshinaga N (1999) PhD Thesis, Ghent University

10. Goux C (1961) Mem Scient Rev Metall 58:661

11. Brandon DG (1966) Acta Metall 14:1479-1484
12. Porter DA, Easterling KE (1992) Phase transformation in metals and alloys. Stanley Thornes Publishers Ltd, UK

13. Saito Y, Mihama K, Uyeda R (1980) Jpn J Appl Phys 19(9): 1603

14. Mackenzie JK, Moore AJW, Nicholas JF (1962) J Phys Chem Solids 23:185

15. Uyeda R (1979) J Crystallagr Soc Jpn 6:113

16. Ranaganathan S, Srivastava AK, Lord EA (2006) J Mater Sci 7696 